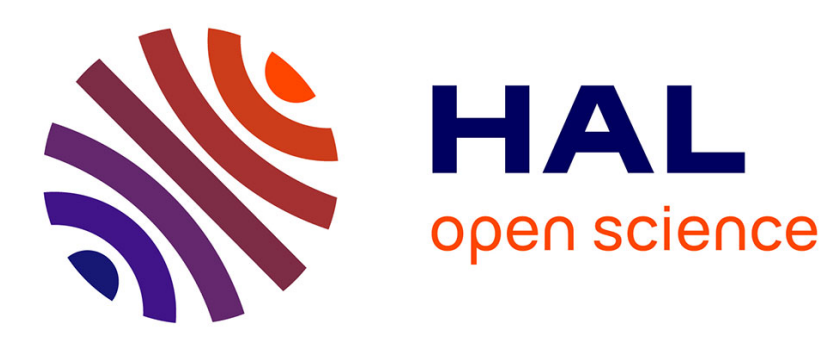

\title{
Vectorial formalism for analysis and design of polyphase synchronous machines
}

\author{
Eric Semail, Alain Bouscayrol, Jean-Paul Hautier
}

\section{To cite this version:}

Eric Semail, Alain Bouscayrol, Jean-Paul Hautier. Vectorial formalism for analysis and design of polyphase synchronous machines. European Physical Journal: Applied Physics, 2003, 22 (3), pp.207220. 10.1051/epjap:2003034 . hal-01099083

\section{HAL Id: hal-01099083 https://hal.science/hal-01099083}

Submitted on 31 Dec 2014

HAL is a multi-disciplinary open access archive for the deposit and dissemination of scientific research documents, whether they are published or not. The documents may come from teaching and research institutions in France or abroad, or from public or private research centers.
L'archive ouverte pluridisciplinaire HAL, est destinée au dépôt et à la diffusion de documents scientifiques de niveau recherche, publiés ou non, émanant des établissements d'enseignement et de recherche français ou étrangers, des laboratoires publics ou privés. 


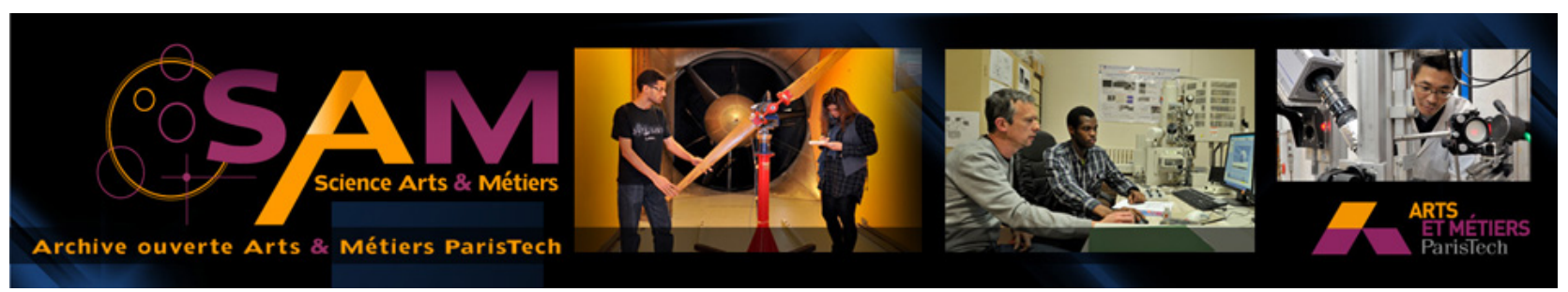

Science Arts \& Métiers (SAM)

is an open access repository that collects the work of Arts et Métiers ParisTech researchers and makes it freely available over the web where possible.

This is an author-deposited version published in: http://sam.ensam.eu

Handle ID: .http://hdl.handle.net/10985/9155

\section{To cite this version :}

Eric SEMAIL, Alain BOUSCAYROL, Jean-Paul HAUTIER - Vectorial formalism for analysis and design of polyphase synchronous machines - The European Physical Journal Applied Physics Vol. 22, n³, p.207-220 - 2003 


\title{
Vectorial formalism for analysis and design of polyphase synchronous machines
}

\author{
E. Semail — A. Bouscayrol, and J.-P. Hautier
}

\begin{abstract}
A vectorial formalism for analysis and design of polyphase synchronous machines without reluctance and saturation effects is described. We prove the equivalence of such a machine with a set of magnetically independent machines, which are electrically and mechanically coupled. Specific problems of polyphase machines can thus be favorably analyzed with this concept. Rules of conception and constraints on electric supply can be deduced. Moreover the vectorial approach, which generalizes the complex phasor method, can also be used to control n-leg Voltage Source Inverters. This methodology is applied to 3-phase and 6phase synchronous machines.
\end{abstract}

\section{Introduction}

The transmission of electric energy by 3-phase networks has led to the development of 3-phase electric machines to ensure electromechanical conversion. These machines have taken benefits of the rise of Digital Signal Processors (DSP) and power semiconductors. In association with power electronics, these 3-phase machines have improved their performances particularly in the field of variable speed drives. Nevertheless, when the power has to be increased, problems appear as much in the inverter as in the machine. The power switches have to commute voltages and currents of higher magnitudes. The partition of the power between numerous phases can be a solution of the problem [25], [43]. Moreover, this kind of structure improves the reliability of the electromechanical conversion [23],[34]. Polyphase machines have been thus industrially developed [30],[16]. The doublestar machines with a $30^{\circ}$ electric phase-shift between the two stars are the most used [4],[15].

The polyphase machines have firstly been supplied by Pulse Amplitude Modulation Current Source Inverter (PAM CSI) [15],[4]. In this case, a machine with $q$ 3-phase stars can be considered as the association of $q 3$-phase machines mechanically coupled on a same shaft. Each 3-phase machine is associated with one of the $q$ stars. This decomposition is possible in spite of the magnetic coupling between the stars, because of a property of the PAM CSI: when there is commutation of a current in a star, the currents in the other stars are constant. Then, there is no interaction between the stars through mutual inductances. They can be considered as magnetically independent.

Nowadays, a Pulse Width Modulation Voltage Source Inverter (PWM VSI) is chosen because of its better dynamic performances. However this voltage supply requires a much more precise modeling of the polyphase machines [48],[33]. The equivalence of a polyphase machine to a set of more simple machines is no more obvious as in the PAM CSI supply. The proposed vectorial approach enables us to show that, with some conditions, it is possible to find an equivalent set of fictitious electric machines. These equivalent machines, called Multimachine system, are mechanically coupled on the same shaft and electrically coupled. The analysis of the fictitious machines gives information for the design of polyphase machines and VSI control [24],[32].

\section{Drawbacks of standard methods}

To study transient or unbalanced states of 3-phase electric machines, different methods have been developed. Matricial and complex phasor methods are the most used. Each one of these two approaches defines a 2-phase fictitious machine, which is mathematically equivalent to the real machine. We examine in this section if it is possible to extend the use of these methods to the study of polyphase machines in transient states.

\subsection{Complex Phasor Method (Space Vector Theory)}

This method consists of working in a complex plane [35],[28]. Consequently, the machine must have a 2dimensional mathematical model. In this case, the various equations can be summarized by only one equation with complex variables. Thus, it is possible to represent this equation in a complex plane. The same concepts have also been developed for steady state: phasor diagrams of A.C. machines [1]. Consequently, the same graphical rules of construction as those developed for steady state can be used in transient states.

Moreover, this method enables an equivalent graphical representation of Voltage Source Inverters (VSI) [22] and Current Source Inverters (CSI) [2],[40]. The time durations of switches can be calculated with a few simple geometrical rules concerning mathematical projections ([22], Fig 1, Fig 2 ). Besides, this inverter modeling allows good control of the converters since third harmonic injection (also called use of zero-sequence component) becomes implicit [12]. The control of multilevel inverters also takes advantage of such a graphical representation for the synthesis of control laws [9]. All these various properties can explain the quick development of the complex phasor method. Nevertheless, one weak point of this approach lies precisely in the fact that modeling in complex plane is only possible with 2dimensional systems.

To carry on the use of this method for an n-dimensional system we must suppose the possibility to decompose it into elementary independent 2-dimensional systems. The problem is to find these elementary systems.

For the analysis of 6-phase or 5-phase machines a few models using two complex planes have been developed [48],[20]. 
The determination of these planes is realized by an inductive approach. The first plane is obtained by considering the first harmonic of magneto-motive force. The second plane is then chosen to be orthogonal to the first one. This approach is possible for 4-dimensional systems but cannot be generalized to n-dimensional systems. The vectorial formalism that we propose leads to the required decomposition into elementary systems.

\subsection{Matricial formalism for n-phase machine study}

In the matricial approach of n-phase electric machines, a vector $n$-space is implicitly considered since vectors with $n$ lines are defined. This space is provided with an orthonormal base $\mathscr{B}_{\mathrm{n}}$ that can be called "natural" since the coordinates of a vector in this base are the measurable values relative to each phase.

In our opinion, the main drawback of the matricial approach

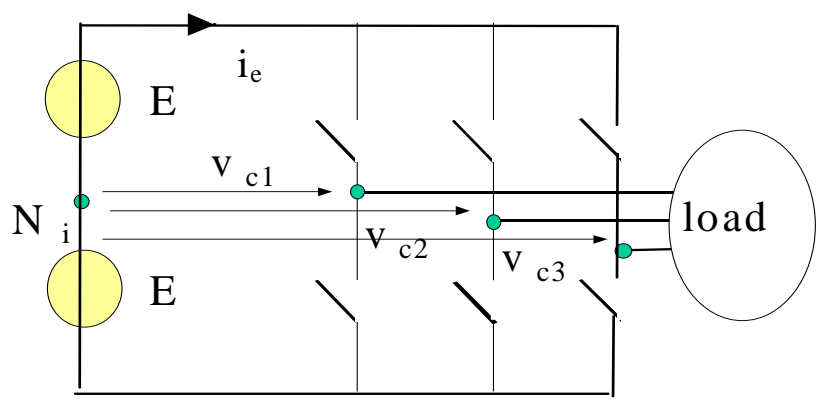

Fig 1: 2-level 3-leg VSI

is to give too much importance to the coordinates of the vectors instead of the vectors themselves. Intrinsic properties

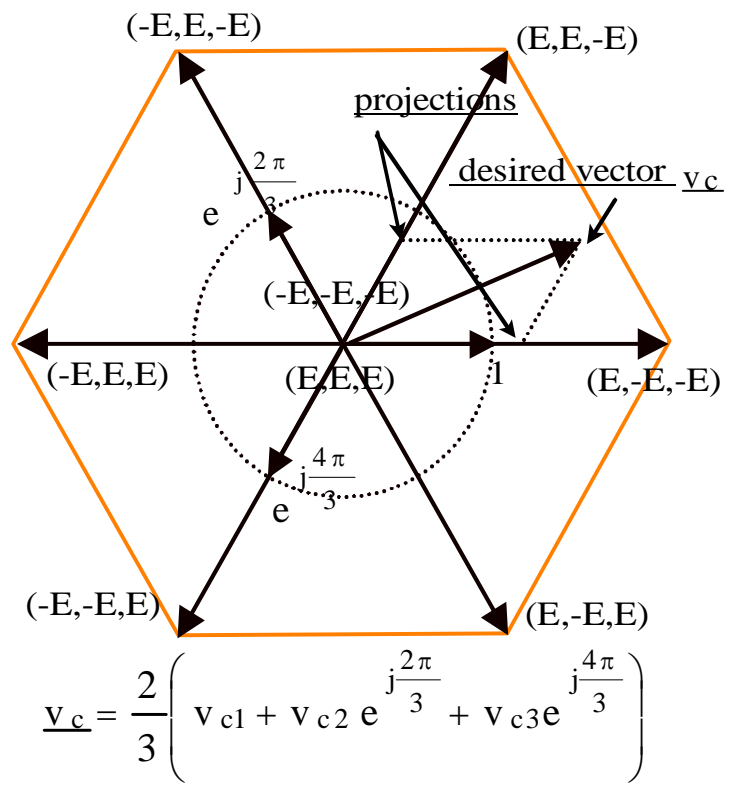

Fig 2: Space phasor representation of 2-level 3-leg VSI. of the vectors and other vectorial mathematical objects (as linear application) are masked and consequently not used. This remark can particularly be applied to the concept of transformation, which has been developed for the study of transient states.

In the matricial method, the emphasis is put on the determination of a transformation matrix, which is a means to get simpler equations. Concordia's, Park's or Fortescue's transformation matrixes allow a simple control of 3-phase machines with delta or star connections. The common way to get a transformation matrix is to find a matricial factorization of the stator self-inductance matrix. With this aim, eigenvalues and associated eigenvectors are determined [1]. A new base $\mathscr{E}_{\mathrm{S}}$ is composed of chosen eigenvectors. It is then possible to express the new decoupled flux equations. As there is an infinity of eigenvectors we can explain thus the multiplicity of transformations that have been developed up to now [29].

From the point of view of the proposed vectorial formalism, a transformation matrix gives only the coordinates, in the "natural" base $\mathscr{B}_{\mathrm{n}}$, of vectors, which constitute a new base $\mathscr{E}_{\mathrm{s}}$ for study. This new base includes only eigenvectors of a characteristic morphism ${ }^{\mathrm{a}} \mathscr{L}_{\mathrm{s}}$ of the electric machine: the coordinates of this morphism $\mathscr{L}_{\mathrm{s}}$ in the "natural" base are the elements of the stator self-inductance matrix. The interest of the concept of morphism is that to a morphism $\mathscr{L}_{\mathrm{S}}$ belongs vectorial entities which are independent of the chosen base: eigenvalues $^{\mathrm{b}}$, eigenvectors and eigenspaces ${ }^{\mathrm{c}}$.

Instead of working with eigenvectors, eigenspaces are preferred. A vector is no more decomposed into coordinates but into a vectorial sum of eigenspace vectors. As the corresponding vectorial decomposition is unique, the simplified vectorial equation is also unique. For ndimensional systems, this approach is particularly interesting since it provides concise vectorial equations.

Moreover, the orthogonality of the eigenspaces of the morphism allows the generalization of the equivalent machine concept. In the matricial formalism, a 2-phase equivalent electric machine (also called 2-axis equations) has already been defined for a 3-phase machine [1]: the torque of the 3-phase machine is produced by this 2-phase fictitious machine. Nevertheless, the matricial formalism, which does not use the eigenspaces, does not lead to the generalization of this concept for polyphase machines. It will be noticed in this paper that the 3-phase machine with usual winding connections is quite a particular case: in general a polyphase machine is equivalent to a set of 1-phase and 2-phase machines.

We can conclude that the matricial approach enables the study of n-dimensional systems but its presentation, which

\footnotetext{
${ }^{\text {a }}$ A morphism is a vectorial linear application.

${ }^{\mathrm{b}}$ If $\lambda$ is an eigenvalue of $\mathscr{L}_{s}$ then there is a vector $\overrightarrow{\mathrm{m}}$ called eigenvector such as $\mathscr{L}(\overrightarrow{\mathrm{m}})=\lambda \overrightarrow{\mathrm{m}}$.

${ }^{c}$ An eigenspace $E_{\lambda}$ is the vector-space generated by the set of eigenvectors associated to an eigenvalue $\lambda$.
} 
uses coordinates of vectors, of morphism (matrix), does not bring out intrinsic vectorial properties.

The proposed vectorial formalism [ 41] will take advantage of vectorial properties: dot product of two vectors (to express power), mixed product (to determine activation time of VSI switches [38]), morphism and eigenspace (to determine simpler equations), bilinear applications or cross products (to express torque)....

\subsection{Vectorial and tensorial formalisms}

There is another formalism expanded by G. Kron [27], which allows also the definition of mathematical entities with intrinsic properties. Independent of the studying base, these entities are called tensors. This approach is more general than the vectorial one. For example, a morphism can be considered as a particular tensor. We think that a vectorial approach constitutes nevertheless an intermediate step between the tensorial and matricial approaches. The tensorial formalism is all the more interesting as the physical frame used to describe physical phenomenon is not orthonormal (for example atomic positions of a crystal in vibration). In this case, the choice of non-orthonormal base in a non-Euclidian vector-space allows us to keep the equations simple.

For the study of electric machines the use of non-orthonormal base is not common and not useful except perhaps for reluctance machines [ 44]. A vectorial formalism in Euclidian vector space is sufficient to introduce the concept of the Multi-Machine that we propose for polyphase machines. G. Kron has already introduced this kind of concept for 3-phase machine to take into account the space harmonics [26].

\section{Generalization of transformation concept}

In order to show that a polyphase machine is equivalent to a set of 1-phase and 2-phase machines, we have to bring out vectorial properties of the stator self inductance matrix. The analysis of its properties enables the generalization of the transformation concept. Of course, the transformation matrixes which generalize Park's and Concordia's transformations for a n-phase machine have already been defined [29],[36] and used, [14],[46],[13],[20],[37] in particular cases. Our formalism defines a larger class of systems for which these transformations can be used. This is possible because, in the vectorial approach, transformations are only the expression of vectorial properties linked to the stator inductance matrix.

At first, an Euclidian vector n-space is associated with an nphase machine. Then we consider that the stator inductance matrix is the characterization, in a natural base, of a linear application also called an endomorphism ${ }^{\mathrm{d}}$.

In the next paragraphs, we give some of its properties and then we use them for two kinds of machines: a 3-phase and a 6-phase machine.

\footnotetext{
d An endomorphism is a morphism inside a same vector space.
}

\subsection{Endomorphism and stator inductance matrix}

Let us consider the stator inductance matrix $\left[L_{s}^{n}\right]$ of a polyphase machine. We interpret it as the matrix of an endomorphism $\mathscr{L}_{\mathrm{s}}$ in an orthonormal base $\mathscr{B}_{\mathrm{n}}$ classified as "natural”. This endomorphism $\mathscr{L}_{\mathrm{s}}$ has properties independent of the choice of the studying base: eigenvalues, eigenvectors and eigenspace. To get them we have only to examine $\left[L_{s}^{n}\right]$.

As mutual inductance between two windings $r$ and $s$ are identical $\left(\mathrm{M}_{\mathrm{rs}}=\mathrm{M}_{\mathrm{sr}}\right)$ then the matrix is symmetrical. This symmetry implies the existence of a base of eigenvectors. Moreover the eigenspaces of $\mathscr{L}_{s}$ are orthogonal each other and the dimension of an eigenspace $E_{\lambda}$ is equal to the multiplicity order $^{f}$ of the associated eigenvalue $\lambda$. For example, if the order of multiplicity is one (respectively two), the eigenspace is a vectorial line (respectively vectorial plane).

To obtain an orthonormal base of eigenvectors we have only to choose in each eigenspace an orthonormal base. The classic transformation matrixes of Park or Concordia are nothing else than tables that allow us to find the coordinates of these eigenvectors.

If the order of multiplicity of all eigenvalues is one, then there is only one orthonormal base of eigenvectors. Consequently, only one transformation that keeps the power invariant can then be elaborated.

On the other hand, if the order of multiplicity is not one for one eigenvalue, then there is an infinity of orthonormal bases of eigenvectors. Consequently an infinity of transformations keeping invariant the power can be defined. This property explains the great number of transformations that have been proposed in the past.

In the following examples, we study $\mathscr{L}_{\mathrm{s}}$ and obtain simplified vectorial flux equations.

\subsection{Example of a 3-phase machine}

To highlight the particularity of the proposed approach, the well-known 3-phase synchronous machine with 2p-pole number is studied. The machine is assumed to be ideal: there is no reluctance effect (uniform air-gap in the machine), no magnetic induced reaction and no saturation effect; the belts are regularly shifted by $120 / \mathrm{p}$ degrees (regular manufacturing).

In a " natural " orthonormal base $\mathscr{B}_{\mathrm{n}}=\left(\overrightarrow{S_{c 1}}, \overrightarrow{S_{c 2}}, \overrightarrow{S_{c 3}}\right)$, we define the following vectors:

$$
\text { - } \overrightarrow{j_{s}}=j_{s 1} \overrightarrow{S_{c 1}}+j_{s 2} \overrightarrow{S_{c 2}}+j_{s 3} \overrightarrow{S_{c 3}} ;
$$

$\left(\mathrm{j}_{\mathrm{sk}}\right.$ stator current in the phase no. $\mathrm{k}$ )

\footnotetext{
e Defined in paragraph 2.2

${ }^{\mathrm{f}}$ The order of multiplicity of an eigenvalue $\lambda$ is equal to the number of times this eigenvalue is root of the $\mathscr{L}_{s}$ characteristic polynomial.
} 


$$
\text { - } \overrightarrow{\phi_{s}}=\phi_{s 1} \overrightarrow{S_{c 1}}+\phi_{s 2} \overrightarrow{S_{c 2}}+\phi_{s 3} \overrightarrow{S_{c 3}}
$$

( $\phi_{\mathrm{sk}}$ linked flux of the stator phase no. $\mathrm{k}$ ).

The hypothesis allows us to express the stator inductance matrix as:

$$
\left[L_{s}^{3}\right]=\left[\begin{array}{ccc}
L_{s s} & M_{s s} & M_{s s} \\
M_{s s} & L_{s s} & M_{s s} \\
M_{s s} & M_{s s} & L_{s s}
\end{array}\right]
$$

The solution of the characteristic equation $\operatorname{det}\left(\left[L_{s}^{3}\right]-\lambda\left[I_{3}\right]\right)=0$ gives two eigenvalues:

$$
L_{0}=L_{s s}+2 M_{s s} \text { and } L_{c}=L_{s s}-M_{s s} .
$$

One of them is double, $L_{c}$, the other single, $L_{0}$. To the last one is associated a 1-dimensional eigenspace $\mathscr{D}$, to the first one $\mathrm{L}_{\mathrm{c}}$ a 2-dimensional eigenspace $\mathscr{R}$.

Let us expand any vector $\vec{x}$ into a sum of two vectors, one per eigenspace. The decomposition, achieved by constructing two orthogonal projections onto the two eigenspaces, gives:

$$
\begin{aligned}
\vec{x} & =\overrightarrow{x_{h}}+\overrightarrow{x_{d q}}, \\
\text { with } \overrightarrow{x_{h}} & \in \mathscr{D} \text { and } \overrightarrow{x_{d q}} \in \mathscr{P} .
\end{aligned}
$$

Former relations between flux and current vectors become then:

$$
\text { - } \overrightarrow{\phi_{s h}}=L_{0} \overrightarrow{j_{s h}}+\overrightarrow{\phi_{s r h}}
$$$$
\text { - } \overrightarrow{\phi_{s d q}}=L_{c} \overrightarrow{j_{s d q}}+\overrightarrow{\phi_{s r d q}}
$$

with $\overrightarrow{\phi_{s r h}}$ and $\overrightarrow{\phi_{\text {srdq }}}$, the respective projections onto $\mathscr{D}$ and $\mathscr{P}$ of $\overrightarrow{\phi_{s r}}$, the flux vector due to the field created by the rotor.

We obtain then a simple vectorial relation between flux and current vectors without the necessity of introducing any matricial transformation. The only necessary concepts are those of eigenvalue, eigenvector and eigenspace.

To establish now the link with the transformation concept, we study the eigenspaces.

For the vectorial line $\mathscr{D}$, the eigenspace associated with $L_{0}$, there is only one normal vector:

$$
\overrightarrow{d_{1}^{c s}}=\frac{1}{\sqrt{3}}\left(\overrightarrow{S_{c 1}}+\overrightarrow{S_{c 2}}+\overrightarrow{S_{c 3}}\right) \text {. }
$$

On the other hand in the plane $\mathscr{P}$, relative to $L_{c}$, there is an infinity of orthonormal bases. We know that this plane is orthogonal to the eigenspace $\mathscr{D}$. The dot product of any vector of $\mathscr{P}$ with $\overrightarrow{d_{1}^{c s}}$ is then equal to zero. We easily obtain the equation of the plane $\mathscr{P}$.

$$
x+y+z=0 .
$$

Let us note by $\left(\overrightarrow{d_{2}^{c s}}, \overrightarrow{d_{3}^{c s}}\right)$ an orthonormal base of this plane:

$$
\left\{\begin{array}{l}
\overrightarrow{d_{2}^{c s}}=\sqrt{\frac{1}{3}}\left(x_{2} \overrightarrow{S_{c 1}}+y_{2} \overrightarrow{S_{c 2}}+z_{2} \overrightarrow{S_{c 3}}\right) \\
\overrightarrow{d_{3}^{c s}}=\sqrt{\frac{1}{3}}\left(x_{3} \overrightarrow{S_{c 1}}+y_{3} \overrightarrow{S_{c 2}}+z_{3} \overrightarrow{S_{c 3}}\right)
\end{array} ;\right.
$$

Thus, we obtain the following matricial relations:

$$
\begin{gathered}
{\left[L_{s}^{3}\right]=[P]\left[\begin{array}{ccc}
L_{0} & 0 & 0 \\
0 & L_{c} & 0 \\
0 & 0 & L_{c}
\end{array}\right]\left[P^{t}\right]} \\
\text { with }\left[P^{t}\right]=\sqrt{\frac{1}{3}}\left[\begin{array}{ccc}
1 & 1 & 1 \\
x_{2} & y_{2} & z_{2} \\
x_{3} & y_{3} & z_{3}
\end{array}\right] .
\end{gathered}
$$

The matrix $\left[P^{t}\right]$, whose lines are the coordinates of eigenvectors in the "natural" base $\mathscr{B}_{\mathrm{n}}$, is a passage matrix. It allows expressing the coordinates of a vector in a new base $\mathscr{E}_{\mathrm{S}}$, with those of the "natural" base. It defines a transformation.

We take now the example of Concordia's transformation to show that it is a particular case for which equations (6) and (7) are verified. The following matrix:

$$
\left[P^{t}\right]=\sqrt{\frac{2}{3}}\left[\begin{array}{ccc}
1 / \sqrt{2} & +1 / \sqrt{2} & +1 / \sqrt{2} \\
1 & -1 / 2 & -1 / 2 \\
0 & +\sqrt{3} / 2 & -\sqrt{3} / 2
\end{array}\right],
$$

characterizes the Concordia's transformation (it is the transposed of Concordia's matrix).

Let us note by $\left(x_{h}, x_{d}, x_{q}\right)$ the coordinates ${ }^{\mathrm{g}}$ of a vector $\vec{x}$ in this new base $\mathscr{E}_{\mathrm{s}}$. The relations between flux and current vectors are:

$$
\overrightarrow{\phi_{s}}=L_{0} j_{s h} \overrightarrow{d_{1}^{c s}}+L_{c} j_{s d} \overrightarrow{d_{2}^{c s}}+L_{c} j_{s q} \overrightarrow{d_{3}^{c s}}+\overrightarrow{\phi_{s r}} .
$$

By projection we get:

$$
\left\{\begin{array}{l}
\phi_{s h}=L_{0} j_{s h}+\phi_{s r h} \\
\phi_{s d}=L_{c} j_{s d}+\phi_{s r d} \\
\phi_{s q}=L_{c} j_{s q}+\phi_{s r q}
\end{array}\right.
$$

We find the (6) and (7) vectorial expressions again. These last ones are more general, since they require only the knowledge of the eigenspaces without the definition of a precise base.

\subsection{Example of a 6-phase machine}

We consider a 6-phase synchronous machine with the same assumptions as for the 3-phase machine. As represented in Fig 3, the belts are split into two sets of 3-phase windings, which are spatially phase shifted by 30 electrical degrees. The

$\mathrm{g}$ The index $h$ reminds us that the projection of a vector onto the line $\mathscr{D}$ is classically called the zero-sequence component. 
space harmonics of the magneto-motive force are neglected and the leakage self-inductances have all the same value $\mathrm{L}_{\mathrm{f}}$.

In a "natural" orthonormal base $\mathscr{B}_{\mathrm{n}}=\left(\overrightarrow{S_{C A 1}}, \overrightarrow{S_{C A 2}}, \overrightarrow{S_{C A 3}}\right.$, $\overrightarrow{S_{C B 1}}, \overrightarrow{S_{C B 2}}, \overrightarrow{S_{C B 3}}$ ), we define the following vectors:

- $\overrightarrow{j_{s}}=j_{S A 1} \overrightarrow{S_{C A 1}}+j_{S A 2} \overrightarrow{S_{C A 2}}+j_{S A 3} \overrightarrow{S_{C A 3}}+$

$$
j_{s B 1} \overrightarrow{S_{C B 1}}+j_{s B 2} \overrightarrow{S_{C B 2}}+j_{s B 3} \overrightarrow{S_{C B 3}} \text {; }
$$

- $\overrightarrow{U_{s}}=U_{S A 1} \overrightarrow{S_{C A 1}}+U_{S A 2} \overrightarrow{S_{C A 2}}+U_{S A 3} \overrightarrow{S_{C A 3}}+$

$$
U_{S B 1} \overrightarrow{S_{C B 1}}+U_{s B 2} \overrightarrow{S_{C B 2}}+U_{s B 3} \overrightarrow{S_{C B 3}} \text {; }
$$

- $\overrightarrow{\phi_{s}}=\phi_{S A 1} \overrightarrow{S_{C A 1}}+\phi_{S A 2} \overrightarrow{S_{C A 2}}+\phi_{S A 3} \overrightarrow{S_{C A 3}}+$

$$
\phi_{S B 1} \overrightarrow{S_{C B 1}}+\phi_{S B 2} \overrightarrow{S_{C B 2}}+\phi_{S B 3} \overrightarrow{S_{C B 3}}
$$

With the hypothesis we obtain the following expression of the stator self inductance matrix:

$$
\left[L_{s}^{6}\right]=L\left[\begin{array}{cccccc}
1+\frac{L_{f}}{L} & -\frac{1}{2} & -\frac{1}{2} & +\frac{\sqrt{3}}{2} & -\frac{\sqrt{3}}{2} & 0 \\
-\frac{1}{2} & 1+\frac{L_{f}}{L} & -\frac{1}{2} & 0 & +\frac{\sqrt{3}}{2} & -\frac{\sqrt{3}}{2} \\
-\frac{1}{2} & -\frac{1}{2} & 1+\frac{L_{f}}{L} & -\frac{\sqrt{3}}{2} & 0 & +\frac{\sqrt{3}}{2} \\
+\frac{\sqrt{3}}{2} & 0 & -\frac{\sqrt{3}}{2} & 1+\frac{L_{f}}{L} & -\frac{1}{2} & -\frac{1}{2} \\
-\frac{\sqrt{3}}{2} & +\frac{\sqrt{3}}{2} & 0 & -\frac{1}{2} & 1+\frac{L_{f}}{L} & -\frac{1}{2} \\
0 & -\frac{\sqrt{3}}{2} & +\frac{\sqrt{3}}{2} & -\frac{1}{2} & -\frac{1}{2} & 1+\frac{L_{f}}{L}
\end{array}\right]
$$

Two eigenvalues are found for the endomorphism $\mathscr{L}_{s 6}$ associated with $\left[L_{s}^{6}\right]$ :

$$
L_{c}=3 L+L_{f} \text { and } L_{f} \text {, }
$$

whose orders of multiplicity are respectively two and four.

We associate then to the double eigenvalue $L_{c}$ a vector-plane $\mathscr{P}$ and to $L_{f}$ a 4-dimensional space $\mathscr{H}$.

Let us expand any vector $\vec{x}$ into a sum of two vectors, one per eigenspace. The decomposition, achieved by creating two orthogonal projections onto the two eigenspaces, gives:

$$
\begin{aligned}
\vec{x} & =\overrightarrow{x_{4 h}}+\overrightarrow{x_{d q}}, \\
\text { with } \overrightarrow{x_{4 h}} & \in \mathscr{H} \text { and } \overrightarrow{x_{d q}} \in \mathscr{P} .
\end{aligned}
$$

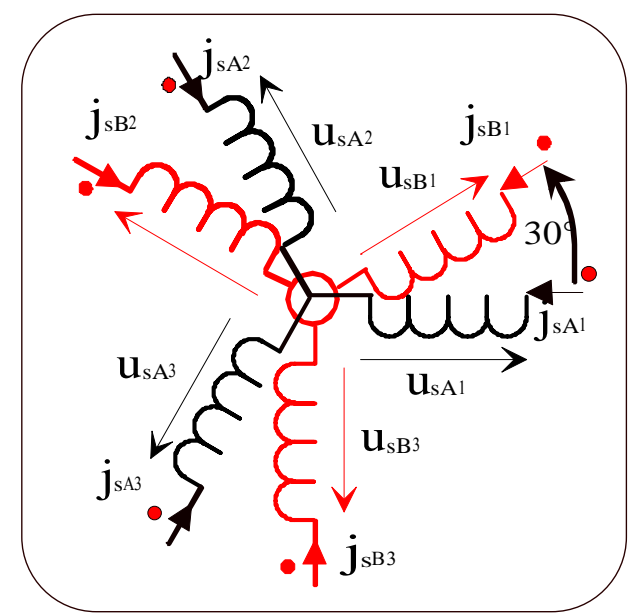

Fig 3: Double-star 6-phase machine

Former relations between flux and current vectors become then:

$$
\begin{aligned}
& \text { - } \overrightarrow{\phi_{s 4 h}}=L_{f} \overrightarrow{j_{s 4 h}}+\overrightarrow{\phi_{s r 4 h}} \\
& \text { - } \overrightarrow{\phi_{s d q}}=L_{c} \overrightarrow{j_{s d q}}+\overrightarrow{\phi_{s r d q}}
\end{aligned}
$$

The equations have been simplified without an explicit choice of a base of eigenvectors. It is quite different from the matricial approach where an explicit transformation has to be chosen to obtain simplified equations.

Now, it is possible to explain why a transformation, which consists of applying twice the 3-phase Concordia's transformation to each star of the double-star machine [11], [33],[3], does not lead to complete decoupling. With this aim, we express this transformation by its characteristic matrix $[T]$, obtained by concatenation of $\left[P^{t}\right]$ (cf. 3.2):

$$
\left[T^{t}\right]=\sqrt{\frac{2}{3}}\left[\begin{array}{cccccc}
1 / \sqrt{2} & 1 / \sqrt{2} & 1 / \sqrt{2} & 0 & 0 & 0 \\
1 & -\frac{1}{2} & -\frac{1}{2} & 0 & 0 & 0 \\
0 & +\frac{\sqrt{3}}{2} & -\frac{\sqrt{3}}{2} & 0 & 0 & 0 \\
0 & 0 & 0 & 1 / \sqrt{2} & 1 / \sqrt{2} & 1 / \sqrt{2} \\
0 & 0 & 0 & 1 & -\frac{1}{2} & -\frac{1}{2} \\
0 & 0 & 0 & 0 & +\frac{\sqrt{3}}{2} & -\frac{\sqrt{3}}{2}
\end{array}\right]
$$

Even if the lines of $T$ define effectively a base of orthonormal vectors, there is still coupling between the equations because the vectors are not eigenvectors of $\mathscr{L}_{s 6}$.

On the other hand, the following matrix $\left[T_{r 6}{ }^{t}\right]$ used in [48], [20],[39], allows the definition of an orthonormal base of eigenvectors: 


$$
\left[T_{r 6}{ }^{t}\right]=\frac{1}{\sqrt{3}}\left[\begin{array}{cccccc}
1 & 1 & 1 & 0 & 0 & 0 \\
1 & -\frac{1}{2} & -\frac{1}{2} & +\frac{\sqrt{3}}{2} & -\frac{\sqrt{3}}{2} & 0 \\
0 & +\frac{\sqrt{3}}{2} & -\frac{\sqrt{3}}{2} & \frac{1}{2} & \frac{1}{2} & -1 \\
0 & 0 & 0 & 1 & 1 & 1 \\
1 & -\frac{1}{2} & -\frac{1}{2} & -\frac{\sqrt{3}}{2} & +\frac{\sqrt{3}}{2} & 0 \\
0 & -\frac{\sqrt{3}}{2} & +\frac{\sqrt{3}}{2} & \frac{1}{2} & \frac{1}{2} & -1
\end{array}\right]
$$

This matrix $\left[T_{r 6}\right]$ plays exactly the same function as Concordia's matrix for 3-phase machines: a decoupling is achieved between stator phases. Each line of $T_{r 6}{ }^{t}$ gives the coordinates, in the natural base, of eigenvectors, which make up an orthonormal base noted $\mathscr{\mathscr { E }}_{\mathrm{s}}=\left(\overrightarrow{d_{1}^{c s}}, \overrightarrow{d_{2}^{c s}}, \overrightarrow{d_{3}^{c s}}, \overrightarrow{d_{4}^{c s}}, \overrightarrow{d_{5}^{c s}}, \overrightarrow{d_{6}^{c s}}\right)$.

With $\left(x_{h A}, x_{d 1}, x_{q 1}, x_{h B}, x_{d 2}, x_{q 2}\right)$ the coordinates of a vector $\vec{x}$ in this base, we obtain finally six equations relative to the stator flux:

$$
\left\{\begin{array} { l } 
{ \phi _ { s h A } = L _ { f } j _ { s h A } + \phi _ { s r h A } } \\
{ \phi _ { s d 1 } = L _ { c } j _ { s d 1 } + \phi _ { s r d 1 } } \\
{ \phi _ { s q 1 } = L _ { c } j _ { s q 1 } + \phi _ { s r q 1 } }
\end{array} \quad \left\{\begin{array}{l}
\phi_{s h B}=L_{f} j_{s h B}+\phi_{s h h B} \\
\phi_{s d 2}=L_{f} j_{s d 2}+\phi_{s r d 2} \\
\phi_{s q 2}=L_{f} j_{s q 2}+\phi_{s q q 2}
\end{array}\right.\right.
$$

We find again, expanded, the vectorial (22) and (23) expressions.

\section{Multimachine Modeling of a Polyphase Machine}

In the above examples it appears, thanks to equations (6) and (7) or (22) and (23), that if the vector $\overrightarrow{\phi_{s r}}$ does not depend on the stator currents then the equations linking flux and stator currents are decoupled inside eigenspaces. So, the stator phases have been magnetically decoupled. It is now possible to define a set of 1-phase and 2-phase machines, which are equivalent to the polyphase machine.

\subsection{Equivalent machine set}

4.1.1 Decomposition into machines without magnetic coupling

To show that the torque of a polyphase machine can be expanded into several torques attached to the eigenspaces, we examine the flow of energy. At first, we express the electric power that is provided to the stator:

$$
p_{s}=\sum_{k=1}^{k=n} u_{s k} j_{s k}=\overrightarrow{u_{s}} \cdot \overrightarrow{j_{s}}
$$

with $u_{s k}$ and $\mathrm{j}_{\mathrm{sk}}$ the stator voltage and current of the phase number $\mathrm{k}$.
Taking into account $R_{s}$, the stator resistance per phase, the stator vectorial differential equation can be put into the following form:

$$
\overrightarrow{u_{s}}=R \vec{j}_{s}+\left[\frac{d \overrightarrow{\phi_{s}}}{d t}\right]_{\mathscr{R}}
$$

The $\mathscr{B}_{\mathrm{n}}$ index reminds that differentiation is operated according to the $\mathscr{B}_{\mathrm{n}}$ natural base.

We obtain then:

$$
p_{s}=\overrightarrow{u_{s}} \cdot \overrightarrow{j_{s}}=R_{s} \overrightarrow{j_{s}} \cdot \overrightarrow{j_{s}}+\left[\frac{d \overrightarrow{\phi_{s}}}{d t}\right]_{\mathscr{\mathscr { h } _ { h }}} \cdot \overrightarrow{j_{s}}
$$

Obviously the first term is relative to stator copper losses. Let us express the second term using the decomposition of $\overrightarrow{\phi_{s}}$ onto the eigenspaces:

$$
\overrightarrow{\phi_{s}}=\sum_{f=1}^{f=F} L_{s f} \overrightarrow{j_{s f}}+\overrightarrow{\phi_{s r f}}
$$

with $\overrightarrow{j_{s f}}$ and $\overrightarrow{\phi_{s r f}}$ the respective projections of $\overrightarrow{j_{s}}$ and $\overrightarrow{\phi_{s r}}$ onto the eigenspace number $\mathrm{f}, \mathrm{L}_{\mathrm{sf}}$ the eigenvalue of the eigenspace and $\mathrm{F}$ the number of eigenspaces.

With the hypothesis of an idealized synchronous machine, the eigenvalues and eigenvectors are constant and $\overrightarrow{\phi_{s r}}$ depends only on the angle $\theta$. So we have:

$$
\left[\frac{d \overrightarrow{\phi_{s}}}{d t}\right]_{\mathscr{\mathscr { h }}}=\sum_{f=1}^{f=F}\left(L_{s f}\left[\frac{d \overrightarrow{j_{s f}}}{d t}\right]_{\mathscr{C}_{s}}+\left[\frac{d \overrightarrow{\phi_{s f f}}}{d t}\right]_{\mathscr{C}_{s}}\right)
$$

with the consequence that $\left[\frac{d \overrightarrow{\phi_{s}}}{d t}\right]_{\mathscr{B}_{n}}$ belongs to eigenspace number $\mathrm{f}$. Therefore, as the eigenspaces are orthogonal to each other, the expression (29) becomes:

$$
\begin{aligned}
& p_{s}=\overrightarrow{u_{s}} \cdot \overrightarrow{j_{s}}=\sum_{f=1}^{f=F}\left(R_{s}\left(\overrightarrow{j_{s f}}\right)^{2}+L_{s f}\left[\frac{d \overrightarrow{j_{s f}}}{d t}\right]_{\mathscr{E}} \cdot \overrightarrow{j_{s f}}+\left[\frac{d \overrightarrow{\phi_{s r f}}}{d t}\right]_{\mathscr{C}} \cdot \overrightarrow{j_{s f}}\right)= \\
& \sum_{f=1}^{f=F}\left(R_{s}\left(\overrightarrow{j_{s f}}\right)^{2}+\frac{d \frac{1}{2} L_{s f}\left(\overrightarrow{j_{s f}}\right)^{2}}{d t}+\left(\frac{d \theta}{d t}\right)\left[\frac{d \overrightarrow{\phi_{s r f}}}{d \theta}\right]_{\mathscr{C}_{s}} \cdot \overrightarrow{j_{s f}}\right)
\end{aligned}
$$

The first term can be considered as stator copper losses, the second term as derivative of stator magnetic energy and the third the product of angular speed $\mathrm{d} \theta / \mathrm{dt}$ and torque:

$$
C_{f}=\left[\frac{d \overrightarrow{\phi_{s r f}}}{d \theta}\right]_{\mathscr{C}_{s}} \cdot \overrightarrow{j_{s f}}
$$

The total torque is then:

$$
C=\sum_{f=1}^{f=F} C_{f}
$$

So we can consider F fictitious machines, each one of them being associated with an eigenspace. These machines are magnetically independent but mechanically coupled through 
the same shaft. For each fictitious machine the number of phases is equal to the dimension of the associated eigenspace. From a total desired torque $C_{r e f}$, the control has to elaborate a reference torque for each fictitious machine. Consequently there is not only one solution. Moreover, there is an electric coupling induced by the change of working base: from the natural base $\mathscr{B}_{\mathrm{n}}$ to the new base $\mathscr{E}_{\mathrm{S}}$. It translates the existing link between real applied voltages and fictitious voltages. So, the problem is to control several machines which are electrically coupled and supplied by only one Voltage Source Inverter [5],[24],[37]. The synthesis of a VSI control law in order to achieve an electric decoupling is nevertheless easier to get than a magnetic one. The reason is that the VSI directly controls electric voltages.

\subsubsection{Representation of the equivalent set (EMR)}

The use of the proposed vectorial formalism leads to the decomposition of a polyphase machine into a set of several machines. For the representation we use the Energetic Macroscopic Representation (EMR) [8] developed for the study of Multimachine Multiconverter Systems (MMS) [5]. This graphical description is based on the causality principle, which allows the definition of inversion rules for the design control laws [6]. Moreover the EMR points out the interactions between the connected power components: principle of action and reaction. Specific coupling devices have been also defined in order to take into account the energy distribution through several conversion chains [5].

On Fig 5 we have the representation of a 3-phase machine supplied by a Voltage Source Inverter (Fig 1). The VSI, electric converter, is represented by three intersected squares since there is only electric coupling. The electric machine is represented by three intersected circles because there is a magnetic coupling: magnetic energy is shared between the three phases.

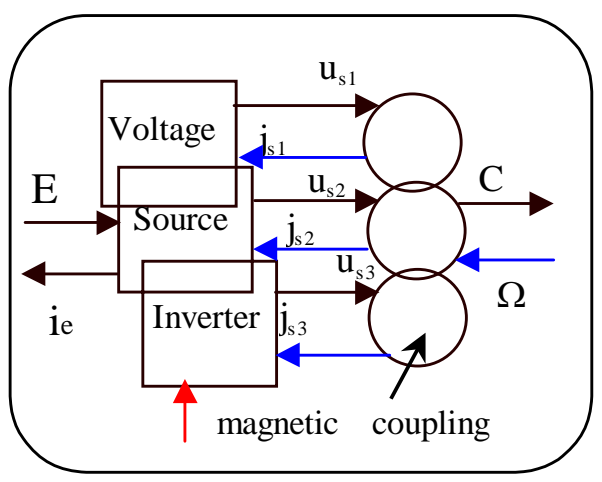

Fig 5: 3-phase machine EMR in natural base

This kind of representation highlights the variables that a system imposes on its environment. They are associated with arrows from outside the system: the torque $\mathrm{C}$ and the currents $\mathrm{j}_{\mathrm{s} 1}, \mathrm{j}_{\mathrm{s} 2}, \mathrm{j}_{\mathrm{s} 3}$ for the machine; the voltages $\mathrm{u}_{\mathrm{s} 1}, \mathrm{u}_{\mathrm{s} 2}, \mathrm{u}_{\mathrm{s} 3}$ and the current $i_{e}$ for the Voltage Source Inverter. The arrows whose directions are towards the inside of a block are associated with variables that are imposed on this system. On Fig 4 and Fig 6 we have given the Energetic Macroscopic Representations of 3-phase and 6-phase machines in the new base $\mathscr{E}_{\mathrm{s}}$. The circles do not intersect any more but there are now intersections between triangles (mechanical couplings). Moreover since there is no more magnetic coupling it is possible to associate explicit Energetic Accumulation Elements (crossed rectangle) to each magnetic subsystem.

In the appendix, we give more precise elements about this Energetic Macroscopic Representation.

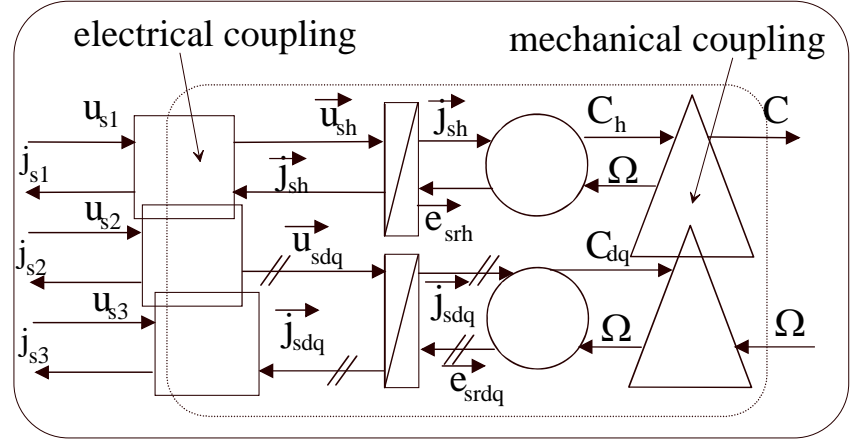

Fig 4: 3-phase machine EMR in the new base $\mathscr{E}_{\mathrm{S}}$

\subsection{Multimachine set of a 3-phase machine}

For a 3-phase machine the expression (28) becomes:

$$
\overrightarrow{u_{s}}=R \overrightarrow{j_{s}}+\left[\frac{d \overrightarrow{\phi_{s}}}{d t}\right]_{\mathscr{\mathscr { R }}}=R_{s} \overrightarrow{j_{s}}+\left[\frac{d \overrightarrow{\phi_{s h}}}{d t}\right]_{\mathscr{\mathscr { R }}}+\left[\frac{d \overrightarrow{\phi_{s d q}}}{d t}\right]_{\mathscr{\mathscr { M }}}
$$

with $\overrightarrow{\phi_{s h}}$ and $\overrightarrow{\phi_{s d q}}$ projections of $\overrightarrow{\phi_{s r}}$ onto the two eigenspaces $\mathscr{P}$ and $\mathscr{D}$ defined in 3.2.

The relations (31) can be expanded into:

$$
\begin{aligned}
& \left\{\left[\frac{d \overrightarrow{\phi_{s h}}}{d t}\right]_{\mathscr{B}}=L_{0}\left[\frac{d \overrightarrow{j_{s h}}}{d t}\right]_{\mathscr{E}_{\mathrm{s}}}+\left[\frac{d \overrightarrow{\phi_{s r h}}}{d t}\right]_{\mathscr{E}}=L_{0}\left[\frac{d \overrightarrow{j_{s h}}}{d t}\right]_{\mathscr{E}_{\mathrm{s}}}+\overrightarrow{e_{s r h}}\right. \\
& \left\{\left[\frac{d \overrightarrow{\phi_{s d q}}}{d t}\right]_{\mathscr{B}_{\mathrm{h}}}=L_{c}\left[\frac{d \overrightarrow{j_{s d q}}}{d t}\right]_{\mathscr{E}_{\mathrm{s}}}+\left[\frac{d \overrightarrow{\phi_{s r d q}}}{d t}\right]_{\mathscr{C}_{\mathrm{s}}}=L_{c}\left[\frac{d \overrightarrow{j_{s d q}}}{d t}\right]_{\mathscr{E}_{\mathrm{s}}}+\overrightarrow{e_{s r d q}}\right.
\end{aligned}
$$

Finally we obtain two decoupled equations:

$$
\left\{\begin{array}{l}
\overrightarrow{u_{s h}}=R_{s} \overrightarrow{j_{s h}}+L_{0}\left[\frac{d \overrightarrow{j_{s h}}}{d t}\right]_{\mathscr{C}}+\overrightarrow{e_{s r h}} \\
\overrightarrow{u_{s d q}}=R_{s} \overrightarrow{j_{s d q}}+L_{c}\left[\frac{d \overrightarrow{j_{s d q}}}{d t}\right]_{\mathscr{C}_{s}}+\overrightarrow{e_{s r d q}}
\end{array}\right.
$$

So, there is effectively no more magnetic coupling but an electric one induced by the change of bases, from $\mathscr{B}_{\mathrm{n}}$ to $\mathscr{E}_{\mathrm{S}}$. A complete equivalent Energetic Macroscopic Representation of a 3-phase machine is given Fig 4. We find two machines electrically and mechanically coupled which are simultaneously controlled. 
The analysis of vectorial equations (37) shows that the machines have electrical time constants, $\tau_{c}=L_{c} / R_{s}$ and $\tau_{0}=L_{0} / R_{s}$, quite different. Effectively, if we consider only the first harmonic of magneto-motive force, we have, taking into account leakage self inductance $L_{f}$ :

$$
L_{s s}=L+L_{f} \text { and } M_{s s}=-L / 2
$$

and so,

$$
L_{0}=L_{s s}+2 M_{s s}=L_{f} \text { and } L_{c}=L_{s s}-M_{s s}=3 L / 2 \text {. }
$$

Consequently, for a machine with little leakage, $\tau_{c}$ is much greater than $\tau_{0}$. To control the current $j_{s h}$, it will be necessary to realize a feedback control with a higher bandwidth than the one necessary for $\overrightarrow{j_{\text {sdq }}}$. If this is not achieved, the supply by VSI induces circulating currents [18].

In fact, it is possible not to supply the 1-phase machine, which has the smallest time constant $\tau_{0}$. We have only to connect the three belts of the machine as usually: a delta-wire connection (then $\mathrm{u}_{\mathrm{s} 1}+\mathrm{u}_{\mathrm{s} 2}+\mathrm{u}_{\mathrm{s} 3}=\mathrm{u}_{\mathrm{sh}}=0$ ) or a star-wire connection without ground (then $\mathrm{j}_{\mathrm{s} 1}+\mathrm{j}_{\mathrm{s} 2}+\mathrm{j}_{\mathrm{s} 3}=\mathrm{j}_{\mathrm{sh}}=0$ ).

We find that a 3-phase machine can generally be considered as equivalent to a 2-phase machine.

In the matricial approach, the concept of a 2-phase equivalent electric machine (also called 2-axis equations) is introduced [1] for one purpose: to solve the equations, which are singular, it is necessary to reduce the dimension of the vector space from 3 to 2 .

In conclusion, the 3-phase machine with usual connections is quite a particular case of polyphase machine since it is equivalent to only one 2-phase machine. This is not the case for other polyphase machines whose control must be thereby more complex.

\subsection{Multimachine set of a 6-phase machine}

We apply exactly the same methodology to a 6-phase machine. Let us consider the voltage vector (Fig 3):

$\overrightarrow{u_{s}}=u_{S A 1} \overrightarrow{S_{C A 1}}+u_{S A 2} \overrightarrow{S_{C A 2}}+u_{S A 3} \overrightarrow{S_{C A 3}}+u_{S B 1} \overrightarrow{S_{C B 1}}+u_{S B 2} \overrightarrow{S_{C B 2}}+u_{S B 3} \overrightarrow{S_{C B 3}}$.

As there is also only two eigenspaces we obtain the following voltage equations:

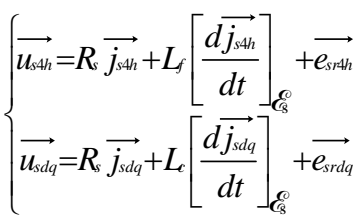

Since the number of phases of each fictitious machine is equal to the multiplicity of the corresponding eigenvalue, we have one 2-phase machine associated with $\mathrm{L}_{\mathrm{c}}$ and one 4phase machine associated with $\mathrm{L}_{\mathrm{f}}$. The EMR formalism gives the representation in Fig 6.

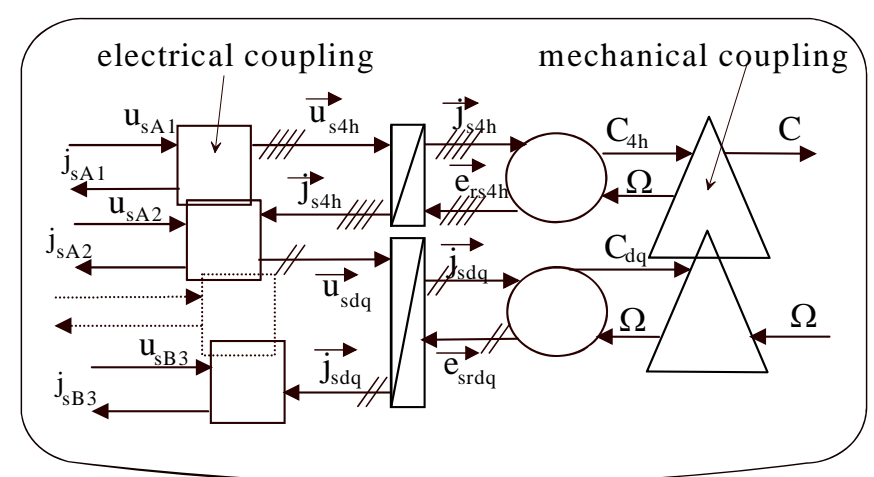

Fig 6: 6-phase machine EMR after magnetic decoupling

We can remark there are still two different time constants with $\tau_{c}>\tau_{0}$. Nevertheless, it is no longer possible to supply the 4-phase machine only by using a wise choice of the connection of belts. If they are connected as in Fig 3, only two components of vector $\overrightarrow{j_{s 4 h}}$ are canceled. When the machine is supplied by a VSI, it is possible to cancel the mean values of the other components but not the instantaneous values. If the period of the pulse width modulation is not small in comparison with $\tau_{c}$,we observe thereby large amplitude currents [33],[16],[17],[24]. These currents do not produce torque when the projection $\overrightarrow{e_{\text {srah }}}$ is equal to zero. So, they can be considered as parasitic currents. How is it possible to reduce and control their amplitude? Further analysis will give us information.

\section{Properties of the Multimachine set}

In this section, we analyse the characteristics of each fictitious machine in order to get design criteria.

\subsection{Fictitious machines and harmonics}

The expression (33) shows that the torque $C_{f}$ is the dot product of the vectorial projections of two vectors. The first one is the stator current vector, which is imposed by control laws. Let us examine the second one that depends on the design of the machine:

$$
\left[\frac{d \overrightarrow{\phi_{s r}}}{d \theta}\right]_{/ \mathscr{B}}
$$

As it is a $2 \pi / p$ periodic function, it can be expanded into Fourier serie and consequently expressed as a sum of vectors associated with harmonic order number k. Properties of symmetry, due to the regular manufacturing assumption, imply, as usual, the cancellation of sine terms and of even cosine terms.

Moreover, when a vector relative to an harmonic is projected onto an eigenspace, then the result is not the same depending on the number of harmonic order. In the following examples, it is notified that, depending on the considered eigenspace associated with the fictitious studied machine, the null terms of the Fourier serie are not the same. There is a distribution of the different harmonics between the eigenspaces. This 
particularity is verified for every vector which has the same mathematical properties as $d \overrightarrow{\phi_{s r}} / d \theta$. Thus, it is possible to associate to each fictitious machine a characteristic family of harmonics.

\subsection{Equivalent machines of a 3-phase machine}

For the 3-phase machine we have two fictitious machines: the first one, associated with a vectorial line $\mathscr{D}$, is a 1-phase machine. The second one, associated with the plane $\mathscr{P}$, is a 2phase machine.

\subsubsection{Examination of $d \overrightarrow{\phi_{s r}} / d \theta$ projections}

As the coordinates of $d \overrightarrow{\phi_{s r}} / d \theta$ are even functions there is no sine terms in the expanded series. So we have:

$$
\begin{gathered}
\frac{d \overrightarrow{\phi_{s r}}}{d \theta}=\sum_{k=0}^{\infty} A_{k} \overrightarrow{H_{k}} \\
\text { with } \\
\overrightarrow{H_{k}}=\cos (k p \theta) \overrightarrow{s_{c 1}}+\cos \left(k p \theta-k \frac{2 \pi}{3}\right) \overrightarrow{s_{c 2}}+\cos \left(k p \theta-k \frac{4 \pi}{3}\right) \overrightarrow{s_{c 3}}
\end{gathered}
$$

Moreover, the coordinates of $\frac{d \overrightarrow{\phi_{s r}}}{d \theta}\left(\theta-\frac{\pi}{2 p}\right)$ are odd functions. Thus the even terms of the Fourier series are nul.

The projection onto the vectorial line $\mathscr{D}$ gives:

$$
\frac{d \overrightarrow{\phi_{s h h}}}{d \theta}=\overrightarrow{d_{c 1}} \sum_{k=1}^{\infty} \frac{A_{k}}{\sqrt{3}}\left\{\cos (k p \theta)+\cos \left(k p \theta-k \frac{2 \pi}{3}\right)+\cos \left(k p \theta-k \frac{4 \pi}{3}\right)\right\}
$$

As $\cos (k p \theta)+\cos \left(k p \theta-k \frac{2 \pi}{3}\right)+\cos \left(k p \theta-k \frac{4 \pi}{3}\right)=0$ for $k \neq 3 q$, then only the triplen harmonics $(k=3 q)$ induce non-null components of $d \overrightarrow{\phi_{\text {srh }}} / d \theta$ :

$$
\frac{d \overrightarrow{\phi_{s r h}}}{d \theta}=\overrightarrow{d_{c 1}} \sum_{q=1}^{\infty} \sqrt{3} A_{3 q} \cos (3 q p \theta)
$$

All the other harmonics are consequently relative to the 2phase machine.

\subsubsection{Criteria of choice for the supply of fictitious machines}

We dispose of two machines to produce the torque of the real machine. To be able to get torque with a machine the projection of $d \overrightarrow{\phi_{s r}} / d \theta$ onto the associated eigenspace must not be equal to zero. Pratically, we have only to achieve a spectral analysis of the electromotive force since it can be remarked that:

$$
\overrightarrow{e_{s r f}}=\left[\frac{d \overrightarrow{\phi_{s r f}}}{d t}\right]_{/ \mathscr{B}_{\mathrm{n}}}=\frac{d \theta}{d t}\left[\frac{d \overrightarrow{\phi_{s r f}}}{d \theta}\right]_{\mathscr{B}_{\mathrm{n}}}=\Omega\left[\frac{d \overrightarrow{\phi_{s r f}}}{d \theta}\right]_{\mathscr{B}_{\mathrm{n}}}
$$

Consequently the 1-phase machine, associated with $\mathscr{D}$, can produce torque only if the electromotive force includes triplen harmonics.

The use of this possibility allows for the increase of the torque for given copper losses [18]. Nevertheless, to supply the 1-phase machine it is necessary to connect the neutral $\mathrm{N}$ point outside: to the $\mathrm{N}_{\mathrm{i}}$ point of the VSI or to a fourth leg (Fig 7).

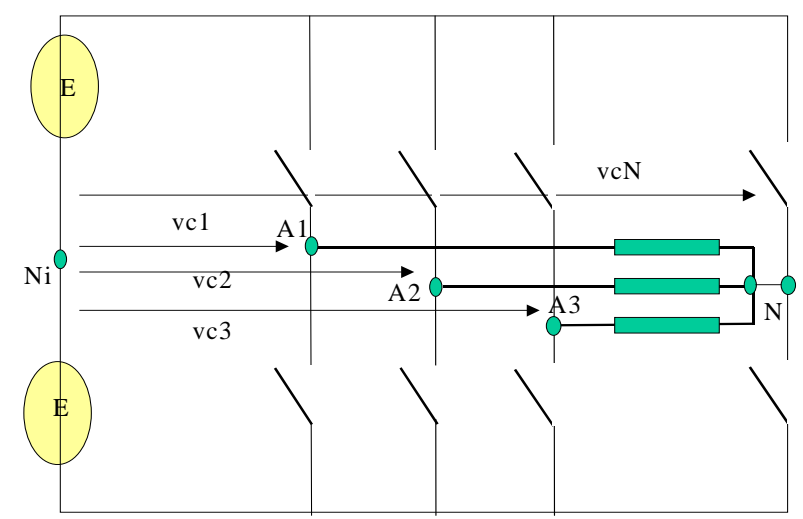

Fig 7: 4-leg VSI to use $3^{\text {rd }}$ order harmonic of electromotive force

In the first case it is necessary to control not only one voltage to a $2 \mathrm{E}$ value but two voltages to $\mathrm{E}$ for the reason that the point $\mathrm{N}_{\mathrm{i}}$ is no more fictitious. In the second case, we have to control a 4-leg VSI.

Besides, as the torque produced by a 1-phase machine is not constant, the use of this machine is not simple if a global constant torque is required. In this case, the 2-phase machine has to supply a variable torque in order to keep constant the global torque. The induced complexity can explain that this kind of structure is not widely used.

On the other hand, if the electromotive forces do not have triplen harmonics, then the supply of this 1-phase machine can only lead to currents that do not produce torque but only losses and ripples.

So, let us examine the necessary conditions to verify the following equation:

$$
\overrightarrow{j_{s h}}=\overrightarrow{0}
$$

As $\mathrm{j}_{\mathrm{sh}}=\left(j_{s 1}+j_{\mathrm{s} 2}+j_{\mathrm{s} 3}\right) / \sqrt{3}$, it appears that a star-wire coupling without neutral is sufficient. Another solution is a delta-wire coupling. We have then $u_{s h}=\left(u_{s 1}+u_{s 2}+u_{s 3}\right) / \sqrt{3}=0$ and consequently $\overrightarrow{j_{s h}}=\overrightarrow{0}$, if there is no electromotive force.

These two kinds of coupling are widely used and induce simplification of the control law of 3-phase machine, which is then equivalent to a 2-phase machine.

In this 2-phase machine we find again all harmonics of electromotive forces except the triplens.

We show now how the use of vectorial formalism enables us to easily find common control laws. 
In case of sinusoidal electromotive force, the vector $d \overrightarrow{\phi_{\text {srdq }}} / d \theta$ has a constant modulus $\left\|d \overrightarrow{\phi_{\text {srdq }}} / d \theta\right\|$.

To get a maximum and constant torque with minimal copper losses $\left(R_{s}\left\|\overrightarrow{j_{s d q}}\right\|^{2}\right)$ we have to impose a vector $\overrightarrow{j_{s d q}}$ of constant modulus, colinear with $d \overrightarrow{\phi_{\text {srdq }}} / d \theta$.

The reason lies in the fact that, for given modulus $\left\|\overrightarrow{j_{s d q}}\right\|,\left\|d \overrightarrow{\phi_{s r d q}} / d \theta\right\|$ of two vectors $\overrightarrow{j_{s d q}}, d \overrightarrow{\phi_{s r d q}} / d \theta$, the dot product, $C=C_{d q}=\overrightarrow{j_{s d q} .} \cdot \overrightarrow{d \phi_{s r d} d} d \theta$, is maximum when the two vectors are colinear. Then the torque $C_{d q}=\left\|\overrightarrow{j_{s d q}}\right\|\left\|d \overrightarrow{\phi_{\text {srdq }}} / d \theta\right\|$ is constant if $\left\|\overrightarrow{j_{s d q}}\right\|$ is also constant.

Indeed, it is possible to extend this result to an electromotive force that contains triplen harmonics.

On the other hand, when $5^{\text {th }}$ and $7^{\text {th }}$ harmonics exist, the control laws become necessarily more complex [19], [10] if constant torque is required. This drawback can lead us to choose polyphase machines to easily reduce torque ripple and the corresponding noise [34],[30].

\subsection{Equivalent machines of a 6-phase machine}

For the considered 6-phase machine, there are two fictitious machines: the first one, associated with the plane $\mathscr{P}$, is a 2phase machine; the second one, associated with the 4dimensional space $\mathscr{H}$, is a 4-phase machine.

\subsubsection{Examination of projections $d \overrightarrow{\phi_{s r}} / d \theta$}

For the same reasons as in 5.2.1, the Fourier serie of $d \overrightarrow{\phi_{s r}} / d \theta$ has only cosine odd terms. So we have:

$$
\begin{gathered}
\frac{d \overrightarrow{\phi_{s r}}}{d \theta}=\sum_{k=1}^{\infty} A_{k} \overrightarrow{e_{t k}}=\sum_{k=1}^{\infty} A_{k}\left(\overrightarrow{e_{t 1 k}}+\overrightarrow{e_{t 2 k}}\right) \\
\text { with } \\
\overrightarrow{e_{t 1 k}}=\sum_{t=1}^{t=3} \cos \left[k p \theta-(t-1) k \frac{2 \pi}{3}\right] \overrightarrow{s_{c t}}
\end{gathered}
$$

and

$$
\overrightarrow{e_{t 2 k}}=\sum_{t=4}^{t=6} \cos \left[k p \theta-k \frac{\pi}{6}-(t-4) k \frac{2 \pi}{3}\right] \overrightarrow{s_{c t}}
$$

The cosine expressions encourage us to work in the complex plane. Let us consider:

$$
\overrightarrow{e_{t 1 k}}=\operatorname{Re}\left\{\overrightarrow{E_{t 1 k}}\right\} \quad \text { et } \overrightarrow{e_{t 2 k}}=\operatorname{Re}\left\{\overrightarrow{E_{t 2 k}}\right\}
$$

with:

- $\overrightarrow{E_{t l k}}=e^{j k p \theta}\left(\overrightarrow{S_{c 1}}+a^{-k} \overrightarrow{S_{c 2}}+a^{-2 k} \overrightarrow{S_{c 3}}\right)$ and $a=e^{j \frac{2 \pi}{3}}$;

- $\overrightarrow{E_{t 2 k}}=b^{k} e^{j k p \theta}\left(\overrightarrow{S_{c 4}}+a^{-k} \overrightarrow{S_{c 5}}+a^{-2 k} \overrightarrow{S_{c 6}}\right)$ and $b=e^{-j \frac{\pi}{6}}$.

If we remark that $a^{3}=1$ and $b^{3}=-j$, we obtain than for $k=3 n$ :

$$
\begin{aligned}
& \text { - } \overrightarrow{e_{t 1 k}}=\sqrt{3} \cos (3 n p \theta) \frac{\left(\overrightarrow{S_{c 1}}+\overrightarrow{S_{c 2}}+\overrightarrow{S_{c 3}}\right)}{\sqrt{3}} \\
& \text { and } \\
& \text { - } \overrightarrow{e_{t 2 k}}=\sqrt{3} \operatorname{Re}\left\{(-j)^{n} e^{j 3 n p \theta}\right\} \frac{\left(\overrightarrow{S_{c 4}}+\overrightarrow{S_{c 5}}+\overrightarrow{S_{c 6}}\right)}{\sqrt{3}}
\end{aligned}
$$

In these two expressions appear $\overrightarrow{d_{1}^{\text {cs }}}$ and $\overrightarrow{d_{4}^{\text {cs }}}$, two eigenvectors of eigenspace $\mathscr{H}$ (see expression (25):

$$
\begin{aligned}
& \text { - } \overrightarrow{e_{t k k}}=\sqrt{3} \cos (3 n p \theta) \overrightarrow{d_{1}^{c s}} \\
& \text { - } \overrightarrow{e_{t 2 k}}=\sqrt{3} \quad \operatorname{Re}\left\{(-j)^{n} e^{j 3 n p \theta}\right\} \overrightarrow{d_{4}^{c s}}
\end{aligned}
$$

So, the triplen harmonics belongs to the subspace $\mathscr{H}$ and more precisely to the plane $\mathscr{R}$ generated by $\overrightarrow{d_{1}^{\mathrm{cs}}}$ and $\overrightarrow{d_{4}^{\mathrm{cs}}}$.

Let us consider now the other harmonics of order $k=3 n \pm 1$ and their projection onto the plane $\mathscr{P}$. As an orthonormal base of this last one is composed of the vectors $\overrightarrow{d_{2}^{\text {cs }}}$ et $\overrightarrow{d_{3}^{\text {cs }}}$, we have to evaluate the dot-product $\overrightarrow{e_{t k}} \cdot \overrightarrow{d_{2}^{c s}}$ and $\overrightarrow{e_{t k}} \cdot \overrightarrow{d_{3}^{c s}}$ :

- $\overrightarrow{e_{t k}} \cdot \overrightarrow{d_{2}^{\mathrm{cs}}}=\frac{\sqrt{3}}{2} A_{k}\left(\cos (k p \theta)+\frac{j^{n} e^{-j k p \theta}+(-j)^{n} e^{j k p \theta}}{2}\right)$

- $\overrightarrow{e_{t k}} \cdot \overrightarrow{d_{3}^{c s}}= \pm \frac{\sqrt{3}}{2} A_{k}\left(\sin (k p \theta)+\frac{(-j)^{n} e^{j k p \theta}-(j)^{n} e^{-j k p \theta}}{2 j}\right)$

We remark that $(j)^{4 u}=1$. When $\mathrm{n}=4 \mathrm{u}$ the previous expressions become:

$\overrightarrow{e_{t k}} \cdot \overrightarrow{d_{2}^{c s}}=\sqrt{3} A_{k} \cos (k p \theta)$ and $\overrightarrow{e_{t k}} \cdot \overrightarrow{d_{3}^{c s}}= \pm \sqrt{3} A_{k} \sin (k p \theta)$ (56)

The harmonics of order $12 u \pm 1$ have thus non-null projections onto the $\mathscr{P}$-plane. Consequently, it is in this plane that we find the projection of the fundamental of the electromotive force. $\mathscr{P}$ is then qualified of "main" plane and also the associated 2-phase machine. We must remark that the 6-phase machine is not equivalent to the "main" machine even if the main part of the torque is produced by the "main" machine.

Let us examine now the properties of the 4-phase machine associated with $\mathscr{H}$. We have already found that the triplen harmonics are projected onto the plane $\mathscr{R}$ generated by $\overrightarrow{d_{1}^{c s}}$ and $\overrightarrow{d_{4}^{c s}}$. Consequently it is possible to decompose the 4phase machine in two 2-phase machines: the first one is associated with $\mathscr{R}$, the second one with an other plane $\mathscr{P}$, subspace of $\mathscr{H}$ and orthogonal to $\mathscr{R}$. In $\mathscr{P}$ we find projections of the last odd harmonics whose orders are $12 u \pm 6 \pm 1$

As the same way as for the 3-phase machine, we qualify of "zero-sequence" the plane $\mathscr{R}$ and the corresponding machine. We find again effectively the zero-sequence components in case of double-star coupling. The plane $\mathscr{P}$ and its corresponding machine is called "secondary". 
We give in Table 1, Table 2 and Table 3, complete results for the projections of $d \overrightarrow{\phi_{s r}} / d \theta$ onto the different planes. We find again the three families of harmonics already pointed out in [25].

Table 1 : projection of $d \overrightarrow{\phi_{s r}} / d \theta$ harmonics onto the "main" plane

\begin{tabular}{|c|c|}
\hline Harmonic order & Main plane $\mathscr{P}$ \\
\hline 1 & $\sqrt{3} A_{1}\left(\cos (p \theta) \overrightarrow{d_{2}^{c s}}+\sin (p \theta) \overrightarrow{d_{3}^{c s}}\right)$ \\
\hline 3 & 0 \\
\hline 5 & 0 \\
\hline 7 & 0 \\
\hline 9 & $\sqrt{3} A_{11}\left(\cos (11 p \theta) \overrightarrow{d_{2}^{c s}}-\sin (11 p \theta) \overrightarrow{d_{3}^{c s}}\right)$ \\
\hline 11 & $\sqrt{3} A_{13}\left(\cos (13 p \theta) \overrightarrow{d_{2}^{c s}}+\sin (13 p \theta) \overrightarrow{d_{3}^{c s}}\right)$ \\
\hline 13 & 0 \\
\hline 15 & 0 \\
\hline 17 & 0 \\
\hline 19 & 0 \\
\hline 21 & $\sqrt{3} A_{23}\left(\cos (23 p \theta) \overrightarrow{d_{2}^{c s}}-\sin (23 p \theta) \overrightarrow{d_{3}^{c s}}\right)$ \\
\hline 23 & $\sqrt{3} A_{25}\left(\cos (25 p \theta) \overrightarrow{d_{2}^{c s}}-\sin (25 p \theta) \overrightarrow{d_{3}^{c s}}\right)$ \\
\hline 25 & \\
\hline
\end{tabular}

Table 2: projection of $d \overrightarrow{\phi_{s r}} / d \theta$ harmonics onto the "zerosequence" plane

\begin{tabular}{|c|c|}
\hline Harmonic order & "zero-sequence" plane $\mathscr{\mathscr { R }}$ \\
\hline 1 & 0 \\
\hline 3 & $\sqrt{3} A_{3}\left(\cos (3 p \theta) \overrightarrow{d_{1}^{c}}+\sin (3 p \theta) \overrightarrow{d_{4}^{c}}\right)$ \\
\hline 5 & 0 \\
\hline 7 & 0 \\
\hline 9 & $\sqrt{3} A_{\theta}\left(\cos (9 p \theta) \overrightarrow{d_{1}^{s}}-\sin (9 p \theta) \overrightarrow{d_{4}^{c s}}\right)$ \\
\hline 11 & 0 \\
\hline 13 & 0 \\
\hline 15 & $\sqrt{3} A_{15}\left(\cos (15 p \theta) \overrightarrow{d_{1}^{c s}}+\sin (15 p \theta) \overrightarrow{d_{4}^{c s}}\right)$ \\
\hline 17 & 0 \\
\hline 19 & 0 \\
\hline 21 & $\sqrt{3} A_{21}\left(\cos (21 p \theta) \overrightarrow{d_{1}^{c s}}-\sin (21 p \theta) \overrightarrow{d_{4}^{c s}}\right)$ \\
\hline 23 & 0 \\
\hline 25 & 0 \\
\hline
\end{tabular}

Table 3: projection of $d \overrightarrow{\phi_{s r}} / d \theta$ harmonics onto the "secondary" plane.

\begin{tabular}{|c|c|}
\hline Harmonic order & Plan $\mathscr{P}_{\mathrm{s}}$ \\
\hline 1 & 0 \\
\hline 3 & 0 \\
\hline 5 & $\sqrt{3} A_{5}\left(\cos (5 p \theta) \overrightarrow{d_{5}^{c s}}+\sin (5 p \theta) \overrightarrow{d_{6}^{c s}}\right)$ \\
\hline 7 & $\sqrt{3} A_{7}\left(\cos (7 p \theta) \overrightarrow{d_{5}^{c s}}-\sin (7 p \theta) \overrightarrow{d_{6}^{c s}}\right)$ \\
\hline 9 & 0 \\
\hline
\end{tabular}

\begin{tabular}{|c|c|}
\hline 11 & 0 \\
\hline 13 & 0 \\
\hline 15 & 0 \\
\hline 17 & $\sqrt{3} A_{17}\left(\cos (17 p \theta) \overrightarrow{d_{5}^{c s}}+\sin (17 p \theta) \overrightarrow{d_{6}^{c s}}\right)$ \\
\hline 19 & $\sqrt{3} A_{19}\left(\cos (19 p \theta) \overrightarrow{d_{5}^{c s}}-\sin (19 p \theta) \overrightarrow{d_{6}^{c s}}\right)$ \\
\hline 21 & 0 \\
\hline 23 & 0 \\
\hline 25 & 0 \\
\hline
\end{tabular}

In Fig 8, we have represented in the EMR formalism the three machines which are mechanically and electrically coupled:

- the "main" machine (mM) ;

- the "zero-sequence" machine (hM) ;

- the "secondary" machine (sM).

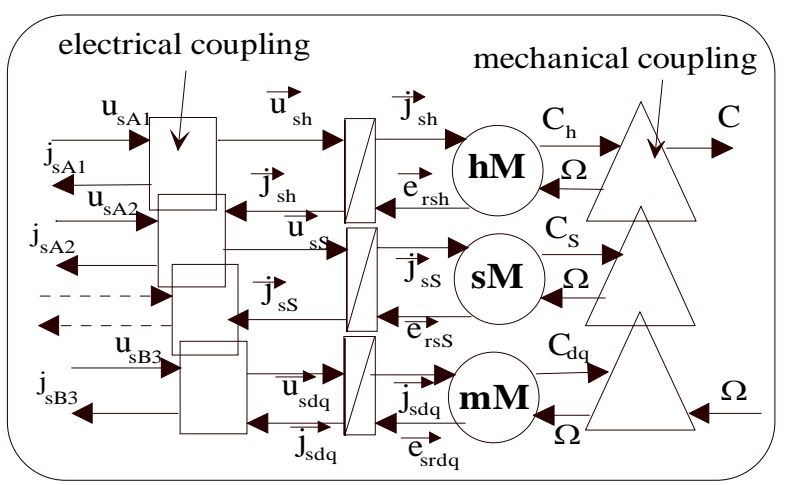

Fig 8: EMR of six-phase machine after magnetic decoupling

5.3.2 Criteria of choice for the supply of the three fictitious machines

We dispose of three machines to produce torque. First, we apply the Multimachine concept to the case of sinusoidal electromotive force. Thus, only the "main" machine will produce torque. The currents in the two other machines are then not desirable since they create only copper losses. They are parasitic currents. To cancel them we must not supply these two machines.

It is quite possible for the "zero-sequence" machine by adapted coupling of the different belts: the more classical one is the double-star coupling represented in Fig 3.

On the other hand, it is not possible to cancel instantaneous currents for the "secondary" machine since the voltages applied to this machine by VSI can not be canceled. Consequently parasitic currents [33], [16], [24] are induced. Mean currents can nevertheless be regulated to zero if null mean voltages are applied thanks to a suitable control law. For the ripple parasitic instantaneous currents, their magnitudes depend on Tmod, the modulation period of the PWM, and on $\tau_{0}$, the electric time constant of the "secondary" machine.

We find that the determination of leakage inductance [39], which is part of $\tau_{0}$, is important. To reduce magnitude of 
parasitic current we must verify: $\tau_{0}>T_{\text {mod }}$. In this paper, we have only considered for the modeling of the 6-phase machine the fundamental of magnetomotive force. We have also neglected mutual leakage inductance. This kind of approach is generally sufficient for the study of a 3-phase machine. It appears that it is no more possible for polyphase machines that are supplied by PWM VSI. To control the value of $\tau_{0}$ the designer has to take into account harmonics of magnetomotive force [24] and mutual leakage inductance [21]. For example to increase $\tau_{0}$ we can favour the fifth harmonic of magnetomotive force by using a new winding distribution.

We have just seen that if the electromotive force is sinusoidal then it would be preferable to supply only the "main" machine. But if the electromotive force possesses harmonics, which are associated with the zero-sequence or secondary machines, then it can be interesting to supply these machines to produce supplementary torque [37],[19],[30],[47]. This kind of possibility has already been studied in [45] for induction machines with concentrated windings. In [31] torque density of a 6-phase machine has been improved by injection of third harmonic currents in the neutral wires of the two stars. For synchronous machine with permanent magnets the designer can also act on the arrangement of the permanent magnets to modify the rate of harmonics.

\section{Conclusion}

The vectorial characterization of the polyphase machines leads to the generalization of the concept of 2-phase

\section{References}

1. Adkins, Hartley R.G. in The general theory of alternating current machines, edited by Chapman and Hall, London, 1975.

2. Amler G., “A PWM Current-Source Inverter for high Quality Drives”, EPE Journal, Vol. 1, no. 1, July 1991, pp. 21-31.

3. Benkhoris M.F., Terrien F., Boucher J.E. “ Numerical Study of a Double Star Synchronous Motor Drive For Electrical Propulsion”, International Conference on Electric Ship, Istanbul (Turkey), September 1998, pp. 70-75.

4. Bhatia R., Krattiger H., Bonanini A., Schafer D., Inge J.T., Sydnor G.H., “Adjustable Speed Drive With A Single 100-MW Synchronous Motor”, ABB Review Issue, No6, 1998, pp.14-20.

5. Bouscayrol A., Davat B., De Fornel B., François B., Hautier J. P., Meibody-Tabar F., Pietrzak-David M., "Multi-machine multi-converter systems: applications to electromechanical drives", EPJ Applied Physics, Vol. 10, equivalent machine commonly used for 3-phase machines. The great difference between 3-phase machines and other polyphase machines has been pointed out. A 3-phase with classical coupling can be considered as a single 2-phase machine. This is not possible with other polyphase machines: at least two 2-phase machines must be taken into account. Thus, the control of a polyphase machine becomes a Multimachine control for which EMR has been developed. Since a polyphase machine can be decomposed into a set of 1-phase and 2-phase machines, it is then possible to apply to each machine the well known method of complex phasors.

As well as the development of models suited to control, this formalism also highlights constraints for the design of polyphase machines. These constraints are mainly due to the presence of secondary and zero-sequence machines. For the designer the association between fictitious machine and a family of harmonics constitutes a powerful and easy means of analysis: magnetomotive and electromotive forces can be modified to get suitable characteristics for the fictitious machines.

In this paper, we have limited the use of the vectorial formalism to a class of synchronous electric machines but the same approach can be considered for induction machines [41]. Besides, it is possible to use at the same time EMR and vectorial formalisms to get a graphic representation of the $\mathrm{m}$ leg VSI which supply these machines [42]. We can then deduce control laws, which enable electric decoupling.

no. 2, May 2000, pp131-147 (common paper of GREEN, L2EP and LEEI, according to the MMS project of GDRSDSE).

6. Bouscayrol A., Davat B., De Fornel B., François B., Hautier J. P., Meibody-Tabar F., Monmasson E., Pietrzak-David M., Razik H., "Control structures for Multi-machine Multi-converter with downstream coupling", EPE'2001, August 2001, Graz (Austria), CDROM, (common paper of GREEN, L2EP, LEEI and LESiR, according to the MMS project of GdR-SDSE).

7. Bouscayrol A., Delarue P., Semail E., François B., Hautier J.P., "Control Method for Multi-leg Voltage Source Inverters" EPE 2001 (European Power Electronics), August 2001, Graz (Austria), CD-ROM.

8. Bouscayrol A., Guillaud X., Hautier J. P., Delarue Ph., "Macro-modeling of electromechanical conversion; application to the electrical machine control", (text in French), RIGE, Vol. 3, no.2, June 2000, pp 257-282.

9. Celanovic N., Boroyevich D., "Fast Space-Vector Modulation Algorithm for Multilevel Three-Phase Converters”, IEEE Transaction Industry Applications, Vol. 37, no.2, March/april 2001, pp 637-641. 
10. Chen S., Song A., Sekiguchi T. "High Efficiency and Low Torque Ripple Control of Permanent Synchronous Motor based on the Current Tracking Vector of Electromotive Force", IEEE-IAS annual meeting 2000, September 2000, Roma (Italy), CD-ROM.

11. Chen Z., Williamson A.C., "Simulation Study of a Double Three Phase Electric Machine", International Conference on Electric Ship, September 1998, Istanbul (Turkey), pp215-220.

12. Chung Dae-Woong, Kim J-S, Sul S-K, “ Unified Voltage Modulation technique for real Time Three-Phase Power Conversion”, IEEE Transaction Industry Applications, Vol. 34, no2, March/April 1998, pp215-220.

13. Coates C.E., Platt D., Gosbell V.J., "Performance Evaluation of a Nine-Phase Synchronous Reluctance Drives”, IEEE-IAS annual meeting 2001, September October 2001, Chicago (USA), CD-ROM,.

14. Gataric S., “ A polyphase cartesian Vector Approach To control of Polyphase AC Machines”, IEEE-IAS annual meeting 2000, October 2000, Roma (Italy), CD-ROM.

15. Godfroi H., Bosc P., “ Large variable speed drives using synchronous motors and frequency converters”, Alstom Review n6, 1986.

16. Gondouin D., Menneron F., "New diesel-electric propulsion system topologies ”, AES2000, October 2000, Paris (France), pp 66-71.

17. Gopakumar K., Ranganathan V.T., Bhat S.R., "Splitphase induction motor operation from PWM voltage source Inverter”, IEEE Transaction Industry Applications, Vol. 29, no.2, September/October 1993, pp 927-932.

18. Grenier D., "Modeling and control strategies of permanent magnet synchronous machines with nonsinusoidal back-electromotive forces", Ph.D. thesis, École Normale Supérieure de Cachan, 1994 (text in French).

19. Grenier D., Yala S., Louis J.P., “ Définition d'extensions de la transformée de Park pour la commande de moteurs synchrones à aimants permanents montés en surface”, European Physical Journal Applied Physics, 1998, no.1 pp233-246.

20. Hadiouche D., Razik H., Rezzoug A., "Modelling of a Double Star Induction Motor for Space Vector PWM Control", ICEM 2000, August 2000, Espoo (Finland) pp392-396.
21. Hadiouche D., Razik H., Rezzoug A., "On The Design of Dual-Stator Windings for Safe VSI Fed AC Machine Drives", IEEE-IAS annual meeting 2001, September October 2001, Chicago (USA), CD-ROM.

22. Holtz J., “Pulsewidth Modulation - A Survey ", IEEE Transactions on Industrial Electronics, Vol 39 no.5, December 1992, pp410-419.

23. Jahns T.M. “ Improved reliability in solid state ac drives by means of multiple independent phase-drive units", IEEE Transaction Industry Applications, Vol. IA-16, May-June 1980, pp 321-331.

24. Kestelyn X., Semail E., Hautier JP. "Vectorial Multimachine modeling for a five phase machine", ICEM 2002, August 2002, Brugges (Belgium), CD-ROM.

25. Klingshirn “ High phase order induction motors _ Part I and II”, IEEE Trans. Power Apparatus Systems, vol PAS -102, 1983, pp 47-59.

26. Kron G. in Equivalent circuits of electric machinery, edited by Dover Edition, $2^{\text {nd }}$ edition, 1966.

27. Kron G. in Tensor analysis of Networks, edited by John Wiley and Sons, London, 1939.

28. Leonhard W. in Control of Electrical Drives, edited by Springer-Verlag, $2^{\text {nd }}$ edition, New York, 1996.

29. Lesenne J., “Contribution à l'étude des composantes relatives et à leur emploi pour l'étude analytique du moteur asynchrone alimenté par des montages à redresseurs ", Thèse de docteur ès Sciences Physique de l’Université des Sciences et Technologies de Lille, 1978.

30. Letellier P., “ High Power Permanent magnet machines for electric propulsion drives ”, AES 2000, October 2000 Paris (France), pp 126-132.

31. Lyra Renato O. C., Lipo Thomas A., “ Torque Density Improvement in a Six-Phase Induction Motor With Third Harmonic Current Injection”, IEEE-IAS annual meeting 2001, September October 2001, Chicago (USA), CDROM,.

32. Martin J. P., Semail E., Pierfederici S., Bouscayrol A., Meibody-Tabar F., Davat B. "Space Vector Control of 5phase PMSM supplied by q H-bridge VSIs" ElectrIMACS 2002, Montreal, August 2002, CD-ROM,.

33. Moubayed N., Meybody-Tabar F., Davat B., "Alimentation par deux onduleurs de tension d'une machine synchrone double étoile”, Revue Internationale de Génie Électrique no.4, 1998, pp 457-470. 
34. Norton P.T., Thompson " The naval electric ship of today and tomorrow", AES 2000, October 2000 Paris (France), pp.80-86

35. Vas P. in Vector control of AC machines, edited by Clarendon Press, 2nd edition, Oxford, 1988.

36. Pasqualini G. "Alimentation par convertisseurs statiques : régimes transitoires”, collection Techniques de l’Ingénieur D3562 Volume D6., 1997.

37. Ruhe S., Toliyat H.A., El-Antably A., "Field Oriented Control of Five-phase Synchronous Reluctance Motor Drive with Flexible 3rd Harmonic Current Injection for High Specific Torque”, IEEE-IAS annual meeting 2001, September October 2001, Chicago (USA), CD-ROM.

38. Semail E. , Rombaut C.,"New method to calculate the conduction durations of the switches in a n-leg 2-level Voltage Source", EPE 2001, August 2001, Graz (Austria), CD-ROM.

39. Semail E., Kestelyn X., "Multi-machine Modeling of a polyphase synchronous machine ", EF'2001

(Électrotechnique du Futur), November 2001, Nancy (France), pp203-208 (text in french).

40. Semail E., M. Amberg, C. Rombaut, “PWM Current Source Inverter: nonlinear control ”, EPF 2000 (Électronique de Puissance du Futur) Lille, November 2000, pp 383-388 (text in French).

41. Semail E., "Tools and studying method of polyphase electrical systems. Generalization of the space vector theory", Ph.D. thesis, University of Sciences and Technologies of Lille, 2000 (text in French).

42. Semail, E., Rombaut C., "New tools for studying voltage-source inverters", IEEE Power Engineering Review, Vol.22, no. 3, March 2002, pp 47-48.

43. Singh G.K., "Multi-phase induction machine drive research-a survey”, Electric Power Systems Research, Vol. 61, 2002, pp139-147.

44. Sturtzer G., Flieller D., Louis J.P, "Extension of the Park Transformation Applied to Non-sinusoïdal Saturated Synchronous Motors”, EPE Journal, Vol. 12, N³, pp. 16-20, Aug. 2002..

45. Toliyat H.A., Lipo T.A., Coleman White J., “ Analysis of a Concentrated Winding Induction Machine for Adjustable Speed Drive Application Part 1 (Motor Analysis)", IEEE Transactions on Energy Conversion, Vol. 6, no.4, 1991, pp679-683.
46. Toliyat H.A., Ruhe S., Huansheng X., “A DSP-Based vector Control of Five-Phase Synchronous Reluctance Motor”, IEEE-IAS annual meeting 2000, October 2000, Roma (Italy), CD-ROM.

47. Toliyat H.A., Waikar S. P., Lipo T.A., " Analysis and Simulation of Five-Phase Synchronous Reluctance Machines Including Third Harmonic of Airgap MMF ”, IEEE Transactions on Industry Applications, Vol. 3 no.2, 1998, pp 332-339.

48. Zhao Y., Lipo A., “ Space Vector PWM Control of Dual Three-Phase Induction Machine Using Space Vector Decomposition ", IEEE Transactions on Industry Applications, Vol 31 no.5, September/October 1995, pp 1100-1109. 


\section{Appendix on EMR formalism}

The Energetic Macroscopic Representation (EMR) is a synthetic graphical tool based on the principle of action and the reaction between elements connected [5], [8]. It leads to a synthetic description of an overall conversion system between two sources (oval pictogram). It uses elements which accumulate energy (rectangular pictogram with an oblique bar) and elements which convert energy without either energy loss nor storage (square for electrical conversion, circle for electromagnetic conversion and triangle for mechanical conversion).

From the EMR of an electromechanical conversion, one can deduce a control structure, which is composed of the maximum of control operations and measurements [8].

This modelling has successfully been applied to electric vehicles, high-speed railway traction systems, wind energy conversion systems, ship propulsion with double-star induction machines.

EMR of an energy conversion - An energy conversion between two sources $S_{1}$ and $S_{2}$ is represented by an association of power components (Fig 9): a conversion element CE and two accumulation elements AE. All of them are connected by exchange vectors.

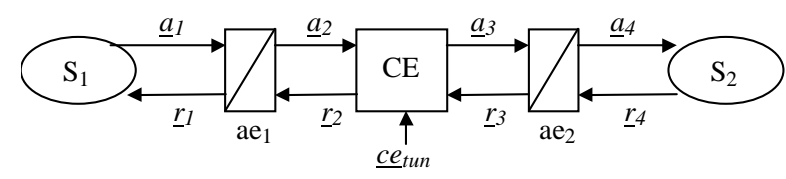

Fig 9 : EMR between two sources

A source element (SE) produces a state variable (output). The source is either a generator or receptor. It is disturbed by the reaction of the connected element (input), for example, the absorbed current for a voltage grid.

A conversion element (CE) yields an energy conversion without energy loss nor storage. Its tuning is ensured by an input vector $e c_{t u n}$ which consumes less power than the transferred one. In some case, there is no tuning input and the conversion transfer is realized with a fixed rate.

An accumulation element (AE) connects an energy source to a conversion element, thanks to an energy storage, which induces at least one state variable. An AE has no tuning input.

The exchange vectors yield the energy to be transferred between the connected components according to the principle of action and reaction. On Fig 9, the source $S_{1}$ is chosen arbitrarily as the upstream source. It produces an action which is transmitted then to the downstream source $S_{2}$ which answers by a reaction. So, one defines a chain of action variables $\left(\underline{a}_{i}\right)$ and a chain of reaction variables $\left(\underline{r}_{i}\right)$. These variables can be scalar or vector. The two connected components are dual each other: if the action is potential, the reaction is kinetic.

Extension to multi-machine multi-converter systems (MMS) - A MMS is composed of several mono-machine monoconverter systems, which share one or more power devices. Thus, it yields energy distribution between electric and mechanical sources through coupled conversion chains, which can yield interactions (perturbations) between power structures. The MMS have to enable best power repartition with lower cost equipment.

The energy distribution is obtained by specific conversion structures [5, 6]. These power components are common to several conversion chains. They are called coupling structures. A coupling conversion structure links an upstream device with many downstream one's, or vice versa. Such structures are drawn by forms with intersections ([Fig 10]).

The electric coupling is associated with electric converters. It corresponds to a common electric device of several converters (power switch, capacitor...). It leads to a common electric variable (voltage, current...).

The magnetic coupling is associated with electric machines. It corresponds to a common part of several machines (winding, magnetic materials...). It leads to a common magnetic variable (flux...).

The mechanical coupling is associated with mechanical converters. It corresponds to a common mechanical device of several converters (shaft...). It leads to a common mechanical variable (speed, force...).

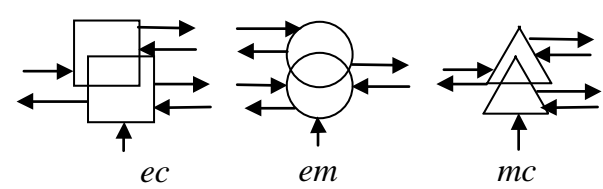

Fig 10: Examples of coupling devices 